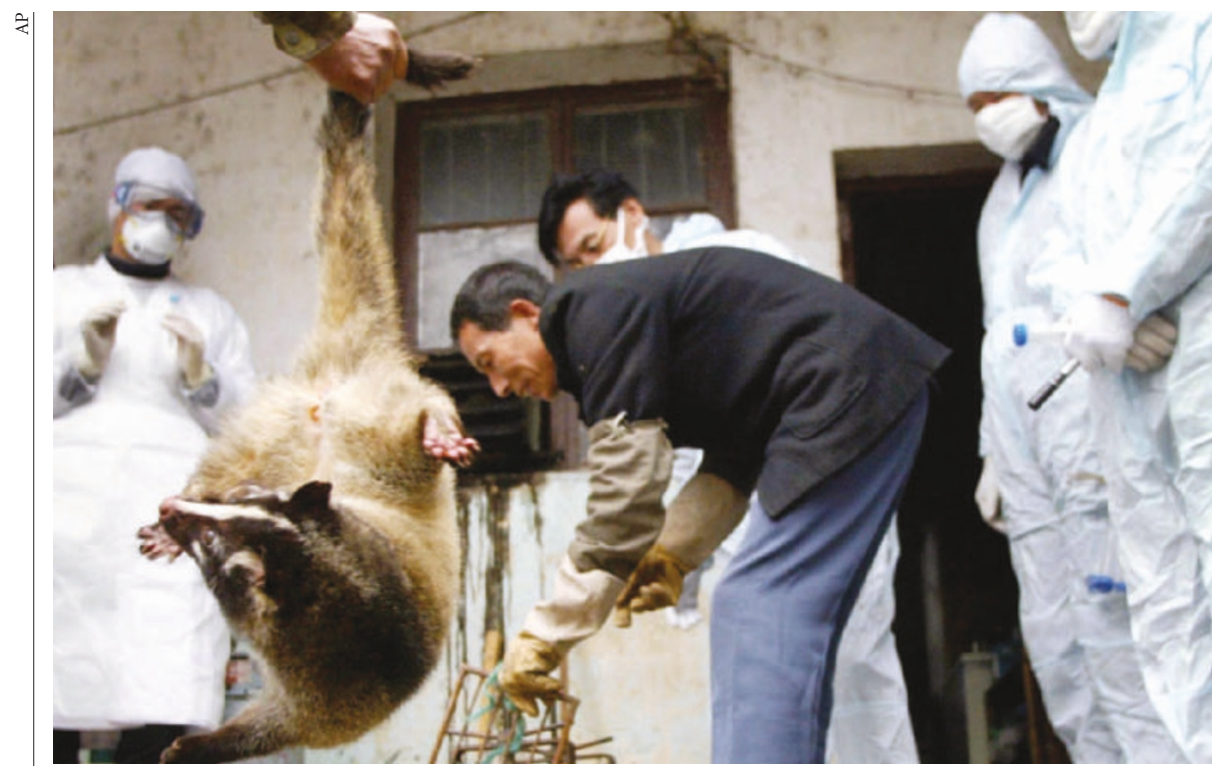

SARS carrier? Animal traders in Guangzhou are handing over masked palm civets for slaughter.

\title{
Antibodies to SARS-like virus hint at repeated infections
}

\section{Helen Pearson}

A virus similar to that responsible for severe acute respiratory syndrome (SARS) infected people in Hong Kong 18 months before SARS reared its head, a recent study says. But some experts say the result is tentative and needs to be confirmed by larger studies.

In a finding published online on 2 January, a team led by Bo Jian Zheng, a microbiologist at the University of Hong Kong, analysed nearly 940 blood samples that had originally been collected in 2001 for a research project on hepatitis $\mathrm{B}$ (B. J. Zheng et al. www.cdc.gov/ncidod/eid/vol10no2/ 03-0533.htm; 2004). SARS first arose in China in November 2002, and public-health experts identified it and alerted the world in March 2003.

In the study, 17 samples carried antibodies either to the human SARS coronavirus, to a closely related animal coronavirus, or to both. Most of those who tested positive had a stronger response to the animal version.

The presence of such antibodies indicates that the Hong Kong inhabitants were exposed to SARS or to one of its animal cousins long before the disease was known or named.Such infections "probably came from a market animal or an environment contaminated by animal viruses," says Zheng.

Experts believe that the 2002 epidemic started when a coronavirus jumped from animals to humans, probably in the live-animal markets of Guangdong province, China. Researchers have found that several market animals and pets, including palm civets and ferrets, can harbour closely related viruses.

The result suggests that the leap from animals to humans was not a one-off event, infectious-disease experts say. A whole family of SARS-like viruses may have been lurking in animals for years, occasionally spilling over into people, says virologist Albert Osterhaus of Erasmus University in Rotterdam, the Netherlands. "There must be other viruses out there," he adds.

In most cases, these coronaviruses probably infect human cells only weakly, perhaps triggering a passing sniffle. Zheng believes that the SARS virus mutated in animals or humans so it can readily jump between people, generating the potentially fatal infection.

The fact that at least one confirmed case of SARS in Guangdong has followed so hot on the heels of last year's outbreak supports the idea that SARS or related viruses can readily move between animals and humans, Zheng believes.

Zheng looked for SARS antibodies in a relatively large, healthy population, and says that similar investigations are under way in China. A 2003 study showed that $40 \%$ of people trading in wild animals in the Chinese markets also carried antibodies to SARS (Y. Guan et al. Science 302, 276-278; 2003).

Some experts caution that the results are not conclusive, however. "I'd like to have seen ten times the number tested," says Christian Drosten of the Bernhard Nocht Institute for Tropical Medicine in Hamburg, Germany.

The researchers now hope to analyse blood from 10,000 healthy people for SARS antibodies. Such studies to flag up hotspots where many people carry antibodies might help scientists to track down the animal source of SARS, says Osterhaus.

\section{India targets local HIV strain in test of AIDS vaccine}

\section{K. S. Jayaraman, New Delhi}

India is gearing up to test an AIDS vaccine targeted at its most prevalent strain of HIV for the first time. Safety trials of the vaccine are expected to begin this summer.

With 60,000 AIDS deaths confirmed so far and an estimated 4.5 million infections, India is second only to South Africa as the country hardest hit by AIDS. About 30 AIDS vaccine trials are already under way worldwide, and the first Indian trial may go some way to address concerns that India has been slow to respond to the epidemic.

"There have been no vaccine trials in India, and that's obviously a real worry," says Seth Berkley, president of the International AIDS Vaccine Initiative (IAVI), a non-profit organization based in New York that is helping to organize the trial. "This is critically important, not only to India, but to the global effort to find a vaccine."

The National AIDS Research Institute in Pune in western India is hoping to conduct the phase I trial, which is only intended to establish the safety of the vaccine, on $\mathbf{4 0}$ healthy volunteers in June.

India has in the past rejected plans to test imported vaccines as they did not target HIV subtype $C$, which is prevalent in India. Early vaccine candidates had been aimed instead at subtype $B$, which is prevalent in America and Europe.

Since 2000, the Indian government has been working with IAVI to develop and evaluate an India-specific AIDS vaccine. "This vaccine is now ready," says Sekhar Chakrabarti, a scientist at the Indian Council for Medical Research who is involved in its development.

The vaccine to be tested is a live attenuated modified vaccinia Ankara virus containing the coding sequence of six $\mathrm{HIV}-1$ subtype $\mathrm{C}$ genes isolated from an Indian strain. For the phase I trial, it will be produced by Therion Biologics, a biotech company based in Cambridge, Massachusetts.

Some AIDS groups have attacked the plan for the safety trial, which has yet to be approved by regulators. "We should abandon the plan, as there is no track record of success by previous candidate vaccines," says Ishwar Gilada, secretary general of the Peoples Health Organization, an AIDS-prevention group in Mumbai. But Chakrabarti says that the vaccine candidate has a greater chance of success than older vaccines. 